\title{
Um estudo da viabilidade de um programa de seguro agrícola baseado em um índice de produtividade regional em Castro (PR)*
}

Vitor Augusto Ozaki**

Ricardo Shirota***

Resumo: Este trabalho analisa a viabilidade de um seguro agrícola baseado em um índice de produtividade regional na região de Castro, no Estado do Paraná. Em particular estimam-se os seguintes indicadores: redução do risco sistêmico proveniente da aquisição deste tipo de contrato, o nível de cobertura ótimo, a correlação entre a produtividade regional e a produtividade individual, e o impacto do nível de agregação da produtividade nos indicadores relacionados. A análise empírica foi realizada para milho e soja, no período de 1990 a 2002, com dados municipais, e de 1994 a 2003, com dados individuais. Os resultados mostraram que o seguro regional poderia ser utilizado como uma alternativa plausível na região, visto que a maioria dos produtores se beneficiariam de uma relativa redução do risco sistêmico.

Palavras-chave: seguro agrícola, risco sistêmico, correlação temporal Classificação JEL: Q19

Abstract:This article studies the viability of an area-based crop insurance in Castro county, in the State of Paraná. In particular we estimate the

\footnotetext{
* Os autores agradecem a Marco Antônio Prado da Castrolanda, Willem B. Bouwman, Eltje Jan Loman Filho, Carlos Proença, Rodrigo Tsukahara e Leandro Gimenez da Fundação ABC pelos dados cedidos e a CAPES pelo apoio financeiro.

**Doutor em Economia Aplicada, Pesquisador PRODOC, USP/ESALQ/DEAS. vaozaki@esalq.usp.br

***Professor Doutor e coordenador da Pós-graduação em Economia Aplicada - DEAS/ ESALQ/USP.rshirota@esalq.usp.br
} 
following indexes: reduction of the systemic risk, the optimum level of coverage, the correlation between the regional and the individual yields and the impact of the level of aggregation of yields on the risk reduction. The empirical analysis was carried through for corn and soybean, in the period of 1990 the 2002, with county data and in the period of 1994 the 2003, with individual data. Results shown that area-based crop insurance could be used as a reasonable alternative to manage risk in the region, due to the fact that most producers shown a relative risk reduction.

Key words: crop insurance, systemic risk, temporal correlation

Jel classification: Q19

\section{Introdução}

O seguro agrícola tradicional, ou seja, aquele em que o mecanismo de compensação está atrelado somente à produtividade individual, historicamente, tem apresentado diversos problemas nos países em que foi implantado, como EUA, Espanha, México. (Hazell et al., 1986; Miranda, Skees, Hazell, 1999; Ozaki e Shirota, 2005).

Diversos são os empecilhos para o sucesso do seguro agrícola naqueles países. Dentre eles, destacam-se: o problema do risco moral ${ }^{1}$; a seleção adversa $^{2}$; o risco sistêmico ${ }^{3}$; e, a inexistência de séries suficientemente longas de dados de produtividade agrícola individual. Este último ponto somado à falta de uma metodologia atuarial adequada de precificação de contratos de seguro agrícola, resultam em taxas de prêmio inexatas e mal calculadas com severas implicações para as seguradoras ${ }^{4}$.

\footnotetext{
${ }^{1}$ Situação na qual o segurado decide por tomar certas decisões, que poderiam aumentar a probabilidade de sinistro. Isto acarretaria no aumento do volume das indenizações pagas pela seguradora (Goodwin, 1993).

${ }^{2} \mathrm{~A}$ seleção adversa é a situação em que à medida que o prêmio se eleva, apenas as pessoas que realmente necessitam do seguro adquirem o contrato, selecionando, deste modo, somente aqueles indivíduos mais arriscados na carteira de seguros (Akerlof, 1970).

${ }^{3}$ Quando um evento causador do sinistro ocorre, (por exemplo, uma seca) este não afeta apenas um produtor mas muitos produtores em uma vasta extensão territorial. Tal fenômeno ilustra o que se denomina risco sistêmico ou risco de evento generalizado.

${ }^{4}$ Historicamente, no Brasil, todos os problemas citados ocorrem de maneira severa. Uma ampla revisão sobre a história do seguro agrícola no Brasil, bem como seus principais problemas, podem ser encontrados em Ozaki (2005).
} 
Por outro lado, o seguro de produtividade regional, apresenta uma grande vantagem em relação ao seguro tradicional: a eliminação, total ou parcial, do problema da assimetria de informação e redução dos altos custos associados à fiscalização e à verificação do sinistro, principalmente quando o evento causador do prejuízo atinge uma área relativamente extensa.

As primeiras iniciativas de segurar a lavoura no Brasil ocorreram em meados da década de 1950, com a instituição da Companhia Nacional de Seguros Agrícolas (CNSA), através da Lei n 2.168, de janeiro de 1954. A Companhia operou durante apenas 13 anos, interrompendo suas atividades em 1966, devido aos elevados déficits que não conseguiram ser saneados pelo seu corpo administrativo, principalmente, em função dos problemas já colocados anteriormente (Santos, 1967).

Passados sete anos após a dissolução da CNSA, o governo federal aprovou a Lei $n^{\circ}$ 5.969, de 11 de dezembro de 1973, criando o PROAGRO. No fim da década de 90, após sucessivos anos de déficit, o programa passou por uma reformulação operacional, que possibilitou uma expressiva melhora em seus resultados no período subsequente (Rossetti, 1998; Rossetti, 2001).

Recentemente, a Lei $\mathrm{n}^{\circ} 10.823$, de 19 de dezembro de 2003, concedeu subvenção ao prêmio na aquisição seguro agrícola*. Esta medida tende a incentivar maior demanda de contratos de seguro agrícola pelos produtores no país e tende a popularizar este mecanismo de administração do risco agrícola entre os produtores. Visto que, em função das altas taxas de prêmio, decorrentes do alto risco intrínseco à atividade agrícola, poucos utilizavam o seguro agrícola.

Este artigo analisa a possibilidade da implantação de um seguro baseado em um indicador de produtividade regional ${ }^{5}$. Diversos aspectos pertinentes ao desenho de um contrato de seguro agrícola são discutidos ao longo do trabalho mas, especificamente, ressalta-se o problema do risco sistêmico ${ }^{6} \mathrm{e}$ da correlação existente entre a produtividade regional e municipal.

\footnotetext{
* Em Quintão (2005), Ozaki discute, entre outras coisas, as possíveis implicações do subsídio ao prêmio no mercado de seguro agrícola.

${ }^{5} \mathrm{O}$ seguro baseado em índices de produtividade é largamente difundido nos Estados Unidos, India, Suécia e Canadá (Miranda, Skees e Hazel, 1999) e, atualmente, é ofertado no Brasil no Estado do Rio Grande do Sul.

${ }^{6}$ Um determinado evento climático ocorrido em certo período de tempo que cause danos a uma certa cultura atinge não apenas um produtor, mas toda uma vasta extensão de terra e muitos produtores, de modo que a pressuposição básica para a segurabilidade, que é a independência entre os eventos, é violada. Este tipo de risco é chamado sistêmico.
} 
Um estudo da viabilidade de um programa de seguro agrícola baseado em um índice de produtividade regional em Castro (PR)

\section{Revisão Metodológica}

O seguro baseado em um indicador de produtividade regional foi definido pela primeira vez em Halcrow (1949). Este tipo de seguro teria o prêmio e a indenização baseados na produtividade de determinada área. A indenização seria paga quando a produtividade média da região fosse menor do que um percentual da produtividade normal ou esperada. Esta produtividade seria calculada com base em uma média ponderada da produtividade da região em um determinado período de tempo, levando em conta as mudanças econômicas e tecnológicas, que poderiam influenciar na variação da produtividade. O limite geográfico da região, sua uniformidade e homogeneidade e a correlação entre a produtividade dos produtores segurados com a da região considerada, são alguns aspectos importantes neste tipo de seguro.

No modelo de Miranda (1991), a produtividade individual é decomposta em um componente sistêmico ${ }^{7}$, que são os fatores que afetam os produtores em uma determinada área, e um componente não sistêmico. Deste modo, a produtividade local do produtor $i, \widetilde{y}_{i}$, pode ser caracterizada, da seguinte maneira:

$$
\tilde{y}_{i}=\mu_{i}+\beta_{i}(\tilde{y}-\mu)+\tilde{a}_{i}
$$

Em que: $\beta_{i}$ representa a sensibilidade da produtividade individual aos fatores sistêmicos que afetam a produtividade regional; $\mu_{\mathrm{i}}$ é a média da produtividade individual; $\mu$ é a média da produtividade regional; $\widetilde{y}$ é a produtividade regional, o termo $\beta_{i}(\widetilde{\mathbf{y}}-\mu)$ o risco sistêmico; e, $\tilde{a ̆}_{\mathrm{i}} \mathrm{o}$ risco não sistêmico.

Desta forma, um contrato de seguro de produtividade regional, cobra

${ }^{7}$ O componente sistêmico ou não diversificável ocorre devido ao fato do prejuízo ao nível do produtor conter uma alta correlação espacial, ou seja, um evento climático extremo, como por exemplo, uma geada que poderia afetar uma grande quantidade de produtores em uma vasta extensão de território. Este evento generalizado se traduz na inexistência de independência entre os eventos, conseqüentemente, elevando o risco de uma seguradora administrar uma carteira agrícola. Tendo a seguradora que se responsabilizar pelo risco adicional, o aumento da taxa do prêmio é inevitável, caso não exista a possibilidade da transferência deste risco para um mercado ressegurador nacional ou internacional ou através do mercado financeiro. 
um prêmio $P$, e paga uma indenização ${ }^{8}$ igual a $\left(y_{c}-\tilde{y}\right)$, caso a produtividade regional $\tilde{y}$ seja menor que a produtividade crítica $y_{c}$. O mecanismo de compensação será dado por $I=\phi \max \left(\mathrm{y}_{\mathrm{c}}-\tilde{\mathrm{y}}, 0\right)$, onde $I$ é a indenização paga, em volume físico, quando o gatilho de compensação representada pela produtividade observada $(\tilde{\mathbf{y}})$ é menor que a produtividade crítica $\left(y_{c}\right)$, também denominada limite do gatilho de compensação, e $\phi$ é o nível de cobertura.

A produtividade líquida individual $\widetilde{\mathrm{y}}_{i}^{\mathrm{L}}$ será dada por $\widetilde{\mathrm{y}}_{\mathrm{i}}^{\mathrm{L}}=\widetilde{\mathrm{y}}_{\mathrm{i}}-P+I$. Isto é, o produtor terá uma produtividade líquida igual à produtividade obtida menos o prêmio pago pelo seguro mais o eventual pagamento da indenização. Supondo que o prêmio seja justo, ou seja, $P=E(I)$, e que o risco de produtividade seja dado pela variância da produtividade líquida, então: $\operatorname{var}\left(\widetilde{\mathrm{y}}_{\mathrm{i}}^{\mathrm{L}}\right)=\operatorname{var}\left(\widetilde{\mathrm{Y}}_{\mathrm{i}}\right)+\operatorname{var}(I)+2 \operatorname{cov}\left(\widetilde{\mathrm{Y}}_{\mathrm{i}}, I\right)$.

Pela aquisição do seguro, o produtor reduz seu risco de produtividade no montante equivalente à diferença entre a variância da produtividade local e a variância da produtividade líquida, dada pela eq. (2):

$$
\Delta_{i}=-\operatorname{var}(I)-2 \operatorname{cov}\left(\tilde{\mathrm{y}}_{\mathrm{i}}, I\right)
$$

$\operatorname{Como} \operatorname{cov}\left(\tilde{\mathrm{y}}, \tilde{\mathrm{a}}_{\mathrm{i}}\right)=0$ e $\operatorname{cov}\left(I, \tilde{\mathrm{a}}_{\mathrm{i}}\right)=0$, de acordo com (1), temos:

$$
\operatorname{cov}\left(\tilde{\mathbf{Y}}_{\mathrm{i}}, I\right)=\beta_{i} \operatorname{cov}(\tilde{\mathrm{y}}, I)
$$

Miranda (1991) define o "beta crítico”, como:

$$
\beta_{c}=-\frac{\operatorname{var}(I)}{2 \operatorname{cov}(\widetilde{y}, I)}
$$

Como a produtividade regional e a indenização são negativamente correlacionadas, então $\beta_{c}>0$. Pode-se verificar, levando em conta a equação (2), (3) e (4) que:

$$
\Delta_{i}=\operatorname{var}(I)\left(\frac{\beta_{\mathrm{i}}}{\beta_{\mathrm{c}}}-1\right)
$$

\footnotetext{
${ }^{8} \mathrm{~A}$ indenização e o prêmio são expressos em unidade físicas, neste modelo e $\mathrm{y}_{\mathrm{c}}=\alpha_{i} \mu$, $\alpha_{i}$ é o percentual de garantia.
} 
Nota-se que, $\beta_{c}$ e a variância da indenização serão determinados pela distribuição da produtividade regional e crítica sendo desta forma, invariantes entre os diversos produtores em uma determinada área. Além disso, a redução do risco de produtividade do produtor ocorrerá somente quando o beta individual exceder o beta crítico.

Introduzindo $\rho_{i}=\operatorname{corr}\left(\widetilde{\mathbf{Y}}_{i}, \widetilde{\mathbf{y}}\right)$, a correlação entre a produtividade individual e a produtividade regional, então $\beta_{i}$ poderá ser escrita como:

$$
\beta_{i}=\rho_{i} \sqrt{\frac{\operatorname{var}\left(\tilde{y}_{i}\right)}{\operatorname{var}(\tilde{y})}}
$$

Percebe-se pela equação acima que, ceteris paribus, quanto menor o risco de base, ou seja, quanto maior a correlacão da produtividade individual com a produtividade regional, maior será o valor de $\beta_{i}$ e, conseqüentemente, maior será a redução do risco propiciado pelo seguro de produtividade regional. Além disso, a redução do risco de produtividade será tanto maior quanto mais elevado for o valor da variância da produtividade individual.

Pela análise até agora realizada, nota-se que a redução do risco é influenciada diretamente pelo beta individual. Para verificar a sensibilidade do $\Delta_{i}$ entre produtores em uma certa área é necessário verificar como o beta individual varia nesta área e como a produtividade crítica afeta o beta crítico.

Seja $w_{i}$ a proporção da área cultivada pelo produtor $i$ na área cultivada total, então $\Sigma_{i} w_{i}=1$ e $\Sigma_{i} w_{i} \widetilde{y}_{i}=\tilde{y}$. Sabendo que:

$$
\sum_{i} w_{i} \operatorname{cov}\left(\tilde{\mathbf{y}}_{\mathrm{i}}, \tilde{\mathbf{y}}\right)=\operatorname{var}(\tilde{\mathbf{y}})
$$

De (7), verifica-se que $\Sigma_{i} w_{i} \beta_{i}=1$, ou seja, teoricamente, a média ponderada dos betas individuais deve ser igual a um.

O beta crítico é uma função crescente da produtividade crítica. Assim:

$0 \leq \beta_{c} \leq 0,5 ; \lim _{y_{c} \rightarrow 0} \beta_{c}=0$; e, $\lim _{y_{c} \rightarrow \infty} \beta_{c}=0,5$.

Por (5) e (8), nota-se que o seguro terá um impacto redutor no risco sempre que $\beta_{i}>0,5$. Na média, o seguro será atrativo para os produtores, pois o $\beta_{i}$ médio dentro de uma determinada área é igual a um. 
Se a produtividade individual for negativamente correlacionada com a produtividade regional, isto é, quando $\beta_{i} \leq 0$, a aquisição do seguro resulta em aumento do risco.

Caso o produtor possa escolher um valor de $\phi$ (maior ou menor do que $100 \%$ ) o nível de cobertura ótimo que maximiza a redução do risco é dado por:

$$
\phi_{i}^{*}=\frac{\beta_{i}}{2 \beta_{c}}
$$

Nota-se que, como $\beta_{c}$ é positivamente relacionado com $y_{c}$, o nível de cobertura se reduz com o aumento de $y_{\mathrm{c}}$ e, também, quando $\beta_{c}$ atinge seu valor máximo. Então, o nível de cobertura atingirá o valor mínimo, ao redor de $\beta_{i}$. Além disso, como o $\beta_{i}$ médio é igual a um, espera-se que o nível de cobertura ótimo tenha um valor maior do que $100 \%$ para grande parte dos produtores.

O nível ótimo de cobertura $\phi_{i}^{*}$ reduzirá o seu risco de produtividade em:

$$
\Delta_{i}^{*}=\rho^{2}\left(\beta_{i}^{2} \sigma_{\tilde{y}}^{2}\right)
$$

Em que: $\rho=\operatorname{corr}(I, \widetilde{\mathbf{y}})$. Lembrando que a variância da produtividade individual é dada por:

$$
\operatorname{var}\left(\tilde{\mathrm{y}}_{\mathrm{i}}\right)=\beta_{\mathrm{i}}^{2} \sigma_{\widetilde{\mathrm{y}}}^{2}+\sigma_{\tilde{\varepsilon}_{\mathrm{i}}}^{2}
$$

Em que, o primeiro termo do lado direito da igualdade corresponde ao componente sistêmico e o segundo ao não-sistêmico. Percebe-se que a redução do risco, dada por (10), é uma proporção $\rho^{2}$ do componente sistêmico, $\beta_{\mathrm{i}}^{2} \sigma_{\tilde{\mathrm{y}}}^{2}$.

$\mathrm{O}$ seguro baseado em um indicador de produtividade regional elimina uma parcela do risco sistêmico enfrentado pelo produtor, mas não elimina o risco não sistêmico.

Outro artigo, posterior ao trabalho de Miranda, analisa outros tipos de contratos e suas implicações na redução do risco e na taxa de prêmio como, por exemplo, em Smith et al. (1994). Mahul (1999), tomando como base o artigo de Miranda (1991), generaliza o modelo em um contexto, 
- Um estudo da viabilidade de um programa de seguro agrícola baseado em um índice de produtividade regional em Castro (PR)

na qual um indivíduo avesso ao risco maximiza a utilidade esperada da produtividade líquida, sujeito a uma função de indenização não negativa e o prêmio como uma função da indenização esperada? .

Miranda (1991) e Mahul (1999) consideraram que a relação entre a produtividade individual $\widetilde{y}_{i}$ e a produtividade regional $\tilde{y}$ segue um modelo linear aditivo (MLA), ou seja, o modelo pode ser entendido como uma regressão linear onde a variável dependente é representada pela produtividade individual e a independente, pela produtividade regional. Além disso, os riscos sistêmico e não-sistêmico são aditivos no modelo.

Mas problemas podem aparecer se a relação entre as produtividades não forem do tipo MLA, consequentemente os resultados encontrados pelos autores podem não ser mais válidos. Neste contexto, Ramaswami e Roe (2004) verificam teoricamente se outros tipos de modelos estruturais podem ser expressos na forma MLA.

Além disso, afirmam que quanto maior o nível de agregação, maior a redução do risco sistêmico e maior será o aumento do risco não-sistêmico. Caso a suposição da existência de um grande número de produtores for relaxada, de modo que se possa considerar um pequeno número de produtores no universo de agregação, então os resultados encontrados usando-se o MLA e a relação linear entre a produtividade individual e regional continuam os mesmos. A diferença é que, neste caso, os estimadores de $\beta_{\mathrm{i}}$ obtidos por mínimos quadrados serão inconsistentes.

Baseados no trabalho de Halcrow (1949) e Miranda (1991), Skees et al. (1997) realizaram um estudo detalhado objetivando a formulação, precificação e implementação de um contrato de seguro baseado em um indicador regional de produtividade.

Embora o problema da assimetria de informação tenha recebido maior atenção na literatura econômica do seguro, o risco sistêmico representa, segundo Miranda e Glauber (1997), a principal causa do fracasso na emergência de um mercado privado de seguro agrícola.

${ }^{9}$ A função de indenização é dada por $I(y) \forall y \in\left[0, y_{\max }\right]$ e o prêmio, $P=\mathrm{c}[\mathrm{E} I(y)]$. Nota-se que $\mathrm{c}(0)=0$ e c' $(I) \geq 0 \forall I(y) \geq 0$. Caso $\mathrm{c}^{\prime}(I)=1$, então define-se o seguro como atuarialmente justo, isto é, a firma seguradora tem como único componente de sua estrutura de custos aqueles destinados ao pagamento da indenização. 
A fim de verificar a possibilidade do seguro baseado em um índice de produtividade regional na região de Castro no Estado do Paraná, foram utilizadas séries de produtividade agrícola de milho e soja para, respectivamente, 26 e 40 produtores, no período de 1994 a $2003^{10}$. Os dados municipais foram liberados pelo Instituto Brasileiro de Geografia e Estatística (IBGE), no período de 1990 a 2002, para milho e soja.

\section{Resultados e discussão}

\subsection{Nível de agregação: "municipal"}

Conforme destacado anteriormente, por Halcrow, o limite geográfico da área, sua uniformidade e homogeneidade e a correlação entre a produtividade em nível de produtor e da área considerada são pontos que merecem destaque no design de um contrato de seguro agrícola regional.

Skees et al. (1997) salientam que para se alcançar a máxima correlação possível entre a produtividade individual e regional, deve-se selecionar o maior número de produtores situados em uma área com características similares de solo e clima.

O limite geográfico da área que irá compor o universo de produtores é um ponto que será abordado com maiores detalhes mais adiante. Na amostra analisada dos produtores, todos se encontravam na área considerada entre boa a regular, dentro de uma escala que vai de boa (1) a desfavorável (10), tendo em vista o potencial agrícola, de acordo com a fertilidade, características físicas e morfológicas do solo e topografia (IBGE, 2004) ${ }^{11}$.

Conforme destacou Miranda, quanto maior a correlação entre a produtividade individual e a regional, maior será o valor de $\beta_{i}$ e, consequentemente, maior a redução do risco proveniente da aquisição de um contrato de seguro agrícola regional. Considerando que o nível de agregação é municipal, o $\beta_{i}$ foi calculado, de acordo, com a equação (6) e a correlação das séries de produtividade foi representada pelo coeficiente de correlação de Pearson, dada por $\rho_{i}=\left(\sum_{i}\left[\widetilde{y}-\mu \rrbracket \tilde{y}_{i}-\mu_{i}\right\rfloor\langle n-1) \sigma_{\widetilde{y}} \sigma_{\widetilde{y}_{i}}\right.$.

\footnotetext{
${ }^{10}$ Fornecidos pela Cooperativa Castrolanda, por intermédio da Fundação ABC.

${ }^{11}$ http://www.ibge.gov.br
} 
Em que $\sigma_{\tilde{y}}$ é o desvio padrão da produtividade regional e $\sigma_{\widetilde{y}_{\mathrm{i}}}$ o desvio padrão da produtividade individual e $n$ o número de observações.

Como pode ser visto, através da eq. 6, o $\beta_{i}$ depende não somente de $\rho_{i}$, mas também da variância da série de produtividade individual. Desta forma, quanto maior $\rho_{i}$ e $\sigma_{\tilde{y}_{\mathrm{y}}}$, maior será o valor de $\beta_{i}$. A Tabela 1 mostra os coeficientes de correlação, $\beta_{i}$ 's, em ordem decrescente, a redução do risco resultante da aquisição do contrato de seguro regional e calculada, a partir da equação (5) e percentual da redução em relação ao risco total. A redução do risco foi calculada para os níveis de produtividade crítica, $\alpha_{i}$ igual a 60 a $120 \%$, em múltiplos de $5^{12}$.

Maiores valores de correlação não significam, necessariamente, maiores valores de $\beta_{i}$, como pode ser visto nos números 17 e 18, na Tabela 1 , à esquerda. $O$ valor de $\beta_{i}$ é afetado também pelo valor da variabilidade da produtividade de cada produtor. Os cinco últimos produtores apresentaram valores de $\beta_{i}$ negativos, na Tabela 1. Isto quer dizer que a cobertura do seguro regional não implica em redução do risco, pelo contrário, significa aumento do risco deste produtor. O mesmo ocorre para os 11 últimos produtores na Tabela 1, à direita.

Produtores que possuem alta correlação com a produtividade regional e alta variabilidade na produtividade poderiam se beneficiar do seguro agrícola regional, pois maior seria a redução do risco sistêmico. Na situação oposta, produtores com baixa correlação e baixa variabilidade não se beneficiariam com tal mecanismo de seguro. Aqueles com correlação negativa seriam prejudicados se demandassem contratos de seguro.

Nas tabelas, nota-se que a aquisição deste contrato de seguro pode ser risco redutor ou risco crescente, para um mesmo produtor, em função do beta crítico $\beta_{c}$, definido pela equação (4). Caso $\beta_{c}$ seja maior do que $\beta_{i}$, então o contrato de seguro será risco crescente. Por exemplo, o beta crítico com $\alpha_{i}=95 \%$ é de 0,1923 . Pela Tabela 1 (esquerda), percebe-se que o $\beta_{i}$ do produtor 1 é igual a 0,2122, maior que o beta crítico. Mas

${ }^{12}$ Em função da série ser relativamente curta, as indenizações dadas por $I=\phi \max \left(\mathrm{y}_{\mathrm{c}}\right.$ - $\tilde{y}, 0$ ), resultaram em valores nulos para todos os anos, ou seja, esta é a situação em que $\tilde{\mathrm{y}}$ é maior do que $\mathrm{y}_{\mathrm{c}}$ em todos os anos, para $\alpha_{i}$ variando de 60 a $90 \%$. Consequentemente, não se define $\beta_{c}$ nem $\Delta_{i}$, nestes casos. 
Tabela 1. Coeficiente de correlação, $\beta_{i}$ e percentual da redução do risco, em relação ao risco total para o milho (à esquerda) e soja (à direita), para dife-

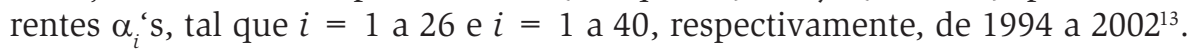

\begin{tabular}{|c|c|c|c|c|c|c|}
\hline \multirow{2}{*}{ Núm. } & \multirow{2}{*}{$\rho_{i}$} & \multirow{2}{*}{$\beta_{i}$} & \multicolumn{4}{|c|}{ Redução do Risco (\%) } \\
\hline & & & 0,95 & 1 & 1,05 & 1,1 \\
\hline 14 & 0,7749 & 1,8267 & 11,4 & 27,6 & 44,0 & 48,8 \\
\hline 18 & 0,3835 & 1,5109 & 3,3 & 7,8 & 12,2 & 13,3 \\
\hline 17 & 0,7160 & 1,3093 & 12,9 & 29,9 & 45,7 & 49,4 \\
\hline 12 & 0,4055 & 1,2284 & 4,4 & 10,0 & 15,1 & 16,2 \\
\hline 22 & 0,2810 & 1,0953 & 2,3 & 5,1 & 7,6 & 8,0 \\
\hline 19 & 0,4219 & 1,0742 & 5,3 & 11,7 & 17,2 & 18,1 \\
\hline 24 & 0,4802 & 1,0672 & 6,8 & 15,2 & 22,3 & 23,5 \\
\hline 16 & 0,6005 & 1,0194 & 11,1 & 24,4 & 35,2 & 36,8 \\
\hline 23 & 0,4615 & 0,9735 & 6,8 & 14,7 & 21,0 & 21,7 \\
\hline 9 & 0,3466 & 0,7723 & 4,5 & 9,0 & 11,6 & 11,2 \\
\hline 2 & 0,2831 & 0,7123 & 3,2 & 6,1 & 7,4 & 6,9 \\
\hline 21 & 0,2554 & 0,6537 & 2,7 & 5,0 & 5,6 & 4,8 \\
\hline 11 & 0,1089 & 0,6289 & 0,5 & 0,9 & 1,0 & 0,8 \\
\hline 3 & 0,2896 & 0,5878 & 3,7 & 6,3 & 6,1 & 4,4 \\
\hline 25 & 0,1127 & 0,5781 & 0,6 & 0,9 & 0,9 & 0,6 \\
\hline 15 & 0,2415 & 0,5478 & 2,7 & 4,2 & 3,5 & 1,9 \\
\hline 5 & 0,3650 & 0,4992 & 6,3 & 8,9 & 5,0 & $-0,1$ \\
\hline 4 & 0,2307 & 0,3947 & 2,7 & 2,0 & $-3,1$ & $-7,3$ \\
\hline 13 & 0,1473 & 0,3830 & 1,1 & 0,7 & $-1,7$ & $-3,5$ \\
\hline 1 & 0,0862 & 0,2122 & 0,1 & $-2,1$ & $-6,8$ & $-9,7$ \\
\hline 10 & 0,1099 & 0,1702 & $-0,4$ & $-7,2$ & $-20,4$ & $-28,1$ \\
\hline 6 & $-0,0252$ & $-0,0840$ & $-1,0$ & $-3,9$ & $-8,5$ & $-10,7$ \\
\hline 26 & $-0,0632$ & $-0,1242$ & $-3,2$ & $-12,3$ & $-26,2$ & $-33,1$ \\
\hline 8 & $-0,0352$ & $-0,1345$ & $-0,9$ & $-3,3$ & $-7,1$ & $-8,9$ \\
\hline 7 & $-0,1296$ & $-0,1970$ & $-6,5$ & $-23,8$ & $-49,3$ & $-61,6$ \\
\hline 20 & $-0,3161$ & $-0,6443$ & $-7,8$ & $-24,3$ & $-46,5$ & $-56,2$ \\
\hline Média & & & 4,6 & 10,0 & 15,4 & 16,6 \\
\hline$\beta_{c}$ & & & 0,1923 & 0,34 & 0,45 & 0,5 \\
\hline
\end{tabular}

${ }^{13}$ A redução de risco máxima foi atingida quando $\alpha_{i}=1,1$ e 1,15, para o milho e soja, respectivamente. 
Um estudo da viabilidade de um programa de seguro agrícola baseado em um índice de produtividade regional em Castro (PR)

\begin{tabular}{|c|c|c|c|c|c|c|c|}
\hline \multirow{2}{*}{ Núm. } & \multirow{2}{*}{$\rho_{i}$} & \multirow{2}{*}{$\beta_{i}$} & \multicolumn{5}{|c|}{ Redução do Risco (\%) } \\
\hline & & & 0,95 & 1 & 1,05 & 1,1 & 1,15 \\
\hline 33 & 0,7275 & 4,1534 & 3,1 & 21,6 & 39,1 & 49,5 & 50,8 \\
\hline 21 & 0,7417 & 2,9738 & 4,4 & 30,4 & 54,3 & 68,1 & 69,8 \\
\hline 25 & 0,5895 & 2,8232 & 3,0 & 20,1 & 35,8 & 44,9 & 45,9 \\
\hline 10 & 0,5895 & 2,6250 & 3,2 & 21,4 & 38,0 & 47,5 & 48,6 \\
\hline 12 & 0,5526 & 2,2875 & 3,2 & 21,1 & 37,2 & 46,3 & 47,3 \\
\hline 32 & 0,4525 & 2,1880 & 2,2 & 14,7 & 25,8 & 32,1 & 32,7 \\
\hline 31 & 0,3701 & 2,0849 & 1,6 & 10,2 & 17,9 & 22,2 & 22,6 \\
\hline 30 & 0,7867 & 1,9709 & 7,4 & 48,4 & 84,5 & 104,2 & 106,2 \\
\hline 38 & 0,4808 & 1,7100 & 3,2 & 20,2 & 34,9 & 42,6 & 43,4 \\
\hline 17 & 0,5156 & 1,2155 & 5,0 & 29,7 & 49,1 & 57,8 & 58,4 \\
\hline 3 & 0,4951 & 1,1585 & 4,8 & 28,2 & 46,3 & 54,1 & 54,5 \\
\hline 36 & 0,5111 & 1,1444 & 5,2 & 30,3 & 49,5 & 57,8 & 58,3 \\
\hline 39 & 0,5258 & 1,1233 & 5,6 & 32,4 & 52,9 & 61,5 & 61,9 \\
\hline 34 & 0,2488 & 1,1005 & 1,3 & 7,4 & 11,9 & 13,8 & 13,9 \\
\hline 15 & 0,1926 & 1,0898 & 0,8 & 4,4 & 7,2 & 8,3 & 8,4 \\
\hline 13 & 0,2224 & 0,8922 & 1,2 & 6,6 & 10,1 & 11,1 & 11,0 \\
\hline 37 & 0,2302 & 0,8001 & 1,4 & 7,3 & 10,8 & 11,4 & 11,3 \\
\hline 26 & 0,3707 & 0,7715 & 3,9 & 19,2 & 28,0 & 29,0 & 28,4 \\
\hline 27 & 0,2872 & 0,7375 & 2,4 & 11,7 & 16,6 & 16,8 & 16,3 \\
\hline 16 & 0,2392 & 0,5390 & 2,2 & 8,0 & 8,3 & 4.6 & 3,5 \\
\hline 11 & 0,2244 & 0,4120 & 2,3 & 5,1 & $-0,4$ & $-9,7$ & $-11,8$ \\
\hline 28 & 0,2921 & 0,3790 & 4,2 & 6,6 & $-7,5$ & $-28,0$ & $-32,6$ \\
\hline 18 & 0,1138 & 0,3348 & 0,7 & 0,3 & $-3,2$ & $-7,7$ & $-8,7$ \\
\hline 19 & 0,0661 & 0,2478 & 0,3 & $-0,9$ & $-4,1$ & $-7,5$ & $-8,1$ \\
\hline 22 & 0,0927 & 0,1757 & 0,6 & $-7,3$ & $-22,8$ & $-38,0$ & $-40,9$ \\
\hline 20 & 0,0497 & 0,1629 & 0,2 & $-2,7$ & $-8,0$ & $-13,2$ & $-14,2$ \\
\hline 24 & 0,0919 & 0,1435 & 0,5 & $-13,2$ & $-38,1$ & $-61,9$ & $-66,3$ \\
\hline 29 & 0,0415 & 0,1218 & 0,1 & $-4,2$ & $-11,7$ & $-18,7$ & $-19,9$ \\
\hline 8 & 0,0075 & 0,0294 & $-0,1$ & $-3,4$ & $-8,5$ & $-12,9$ & $-13,7$ \\
\hline 6 & $-0,0320$ & $-0,0452$ & $-1,8$ & $-33,5$ & $-78,9$ & $-117,4$ & $-124,0$ \\
\hline 2 & $-0,0666$ & $-0,2110$ & $-0,8$ & $-9,7$ & $-21,3$ & $-30,6$ & $-32,1$ \\
\hline 1 & $-0,0979$ & $-0,3408$ & $-0,9$ & $-10,0$ & $-21,3$ & $-30,1$ & $-31,5$ \\
\hline 14 & $-0,1608$ & $-0,5617$ & $-1,3$ & $-13,3$ & $-27,3$ & $-37,8$ & $-39,4$ \\
\hline 7 & $-0,1991$ & $-0,6781$ & $-1,7$ & $-15,8$ & $-32,2$ & $-44,2$ & $-46,0$ \\
\hline 40 & $-0,2445$ & $-1,1698$ & $-1,4$ & $-12,0$ & $-23,6$ & $-31,9$ & $-33,1$ \\
\hline 5 & $-0,4558$ & $-1,4042$ & $-3,9$ & $-33,4$ & $-65,4$ & $-87,7$ & $-90,9$ \\
\hline 23 & $-0,7416$ & $-1,4108$ & $-10,3$ & $-87,9$ & $-172,2$ & $-230,8$ & $-239,3$ \\
\hline 35 & $-0,3869$ & $-1,4176$ & $-2,8$ & $-23,8$ & $-46,6$ & $-62,5$ & $-64,8$ \\
\hline 9 & $-0,3601$ & $-2,4459$ & $-1,4$ & $-11,0$ & $-21,2$ & $-28,0$ & $-28,9$ \\
\hline 4 & $-0,4894$ & $-2,7181$ & $-2,3$ & $-18,1$ & $-34,7$ & $-45,7$ & $-47,3$ \\
\hline Média & & & 2,6 & 17,6 & 32,9 & 39,2 & 39,7 \\
\hline$\beta_{c}$ & & & 0,10 & 0,32 & 0,42 & 0,49 & 0,50 \\
\hline
\end{tabular}

Fonte: Resultados da pesquisa. 
$\operatorname{com} \alpha_{i}=100 \%$, este mesmo $\beta_{i}$ é menor do que o beta crítico $(0,34)$, por isso a redução do risco é negativa para este produtor aos níveis de 100 a $120 \%$.

Além disso, quanto maior o $\beta_{i}$, maior será a redução do risco, para todos os níveis de $\mathrm{y}_{c}$. Percebe-se que o beta crítico se estabiliza no valor máximo de 0,5 , com o aumento da produtividade crítica, conforme já apontava Miranda.

Na escolha ótima, $\phi_{i}^{*}$ será no mínimo $\beta_{i}$ quando $\beta_{c}$ atingir seu valor máximo. Percebe-se, desta forma, que quando $\beta_{c}$ for igual a $0,5, \alpha_{i}=$ 1,1 , na Tabela 1 . O valor de $\phi_{i}^{*}$ para o produtor 14 , por exemplo, é igual a 1,8267 (para este valor de $\alpha_{i}$ ), o mesmo valor de $\beta_{i}$ encontrado na Tabela 1. Este resultado já era esperado, pois conforme ressalta Mahul (1999), se $\beta_{i}>0$, então o nível de cobertura ótimo $\phi_{i}^{*}=\beta_{i}$. Além disso, o nível ótimo de cobertura será inversamente proporcional ao aumento da produtividade crítica.

O nível ótimo de cobertura permite conhecer a máxima redução do risco de produtividade em virtude da aquisição de um contrato de seguro regional. Se o produtor optar pelo nível de cobertura ótimo, então tal redução será igual a 49, 75 e $97 \%$ para $\alpha_{i}=0,95,1,1,05$ e de $100 \%$ para os demais $\alpha_{i}$ 's, no caso do milho e de 29,64 e $90 \%$ para $\alpha_{i}=0,95,1$, 1,05 e de $100 \%$ para os demais $\alpha_{i}$ 's. Pois, percebe-se que a redução do risco, dada pela eq. (10), é uma proporção $\rho=\operatorname{corr}(I, \tilde{y})$ do componente sistêmico, $\beta_{\mathrm{i}}^{2} \sigma_{\widetilde{y}}^{2}$.

Nota-se, desta forma, que quanto maior $\alpha_{i}$, maior será a redução do risco sistêmico e maior o prêmio cobrado pelo seguro. De modo que, ao nível de 1,05, quase todo o risco será eliminado pela aquisição do seguro, nos dois casos. Para níveis maiores do que 1,05, todo o risco é eliminado. O seguro regional elimina certa proporção do risco sistêmico, mas não elimina o risco não sistêmico. O produtor deve administrar este risco remanescente, de modo individual.

Todos os produtores com correlação positiva (Tabela 1) poderiam demandar pelo seguro regional, com diferentes níveis de cobertura e dedutibilidades, mas aqueles com maior correlação e variabilidade na produtividade poderiam ter um benefício maior em função da aquisição deste tipo de seguro. 
Um estudo da viabilidade de um programa de seguro agrícola baseado em um índice de produtividade regional em Castro (PR)

\subsection{Nível de agregação: "cluster"}

Até agora a análise foi realizada utilizando-se o nível de agregação da produtividade regional $\widetilde{y}$ municipal. Conforme aponta Halcrow, o cálculo de $\tilde{y}$ poderia ser realizado através da seleção de 15 a 20 propriedades e o resultado estendido para compreender toda a área. Desta forma, considera-se que $\tilde{\mathbf{y}}=\Sigma_{i} w_{i} \widetilde{\mathbf{y}}_{\mathrm{i}}$, onde $w_{i}$ é a proporção da área cultivada pelo produtor $i$ na área cultivada total, $\Sigma_{i} w_{i}=1$ e $i=1, \ldots, 26$ e $1, \ldots$, 40, para a cultura do milho e soja, respectivamente.

A Tabela 2 mostra a diferença nas produtividades, em relação ao nível de agregação. A produtividade cluster reflete a agregação de 26 produtores de milho e 40 de soja, situados na região de Castro. A produtividade do município corresponde à média municipal. Como pode ser observado na tabela quanto menor o nível de agregação, maior a variabilidade da produtividade.

Tabela 2. Comparação das produtividades (kg/ha) de soja e milho, por nível de agregação municipal e cluster, região de Castro - PR.

\begin{tabular}{lcccc}
\hline \multirow{2}{*}{ Ano } & \multicolumn{3}{c}{ Milho } & \multicolumn{2}{c}{ Soja } \\
\cline { 2 - 5 } & cluster & município & cluster & município \\
\hline 1994 & 8237 & 8700 & 3413 & 3234 \\
1995 & 8745 & 8279 & 3407 & 3182 \\
1996 & 8169 & 8258 & 3259 & 3130 \\
1997 & 7552 & 7921 & 3086 & 2978 \\
1998 & 8395 & 8115 & 3242 & 3027 \\
1999 & 7542 & 7394 & 3163 & 3082 \\
2000 & 8294 & 7948 & 3048 & 3123 \\
2001 & 9658 & 8130 & 3280 & 3271 \\
2002 & 8816 & 8931 & 3440 & 3170 \\
2003 & 8564 & - & 3481 & - \\
\hline Média & 8397 & 8186 & 3282 & 3133 \\
\hline Variância & 383037 & 199356 & 22981 & 8868 \\
\hline
\end{tabular}

Fonte: Resultados da pesquisa.

O fato de se reduzir consideravelmente o nível de agregação fez com que a correlação entre a produtividade individual e a regional aumentasse. A correlação média aumentou de 0,25 , no nível de agregação municipal para 0,51, na agregação cluster.

Além disso, dos 26 produtores de milho, somente 3 apresentaram correlação maior do que 0,5 e 5 produtores tiveram tal coeficiente menor do que 
zero, na Tabela 1, enquanto que 16 produtores apresentaram valor maior do que 0,5 e apenas 2 com coeficiente menor do que zero, na Tabela 3.

No caso da soja, a correlação média aumentou de 0,17 , no caso da agregação municipal, para 0,41, na agregação cluster. Além disso, dos 40 produtores de milho, 9 apresentaram correlação maior do que 0,5 e 11, valores menores do que zero para este coeficiente, na Tabela 1, enquanto que 19 apresentaram valores maiores do que 0,5 e 5, cujo coeficiente de correlação foi menor do que zero, na Tabela 4.

Tabela 3. Coeficiente de correlação, $\beta_{i}$ e redução do risco e percentual da redução do risco, em relação ao risco total para o milho, ao nível de agregação cluster, em 26 fazendas, no período de 1994 a 2003.

\begin{tabular}{|c|c|c|c|c|c|c|c|c|}
\hline \multirow{2}{*}{ Núm. } & \multirow{2}{*}{$\rho_{i}$} & \multirow{2}{*}{$\beta i$} & \multicolumn{6}{|c|}{ Redução do Risco (\%) } \\
\hline & & & 0.95 & 1 & 1.05 & 1.1 & $1.15 \%$ & 1.2 \\
\hline 25 & 0.70 & 2.58 & 7.4 & 14.5 & 21.9 & 26.4 & 30.3 & 30.3 \\
\hline 18 & 0.89 & 2.52 & 12.2 & 24.0 & 36.2 & 43.7 & 50.1 & 50.1 \\
\hline 11 & 0.52 & 2.17 & 4.8 & 9.4 & 14.1 & 17.0 & 19.3 & 19.3 \\
\hline 22 & 0.68 & 1.91 & 9.2 & 17.6 & 26.3 & 31.6 & 35.7 & 35.7 \\
\hline 9 & 0.88 & 1.41 & 19.9 & 36.9 & 53.7 & 64.0 & 70.7 & 70.7 \\
\hline 13 & 0.69 & 1.30 & 13.2 & 24.1 & 34.9 & 41.4 & 45.3 & 45.3 \\
\hline 24 & 0.71 & 1.14 & 15.4 & 27.4 & 39.0 & 46.1 & 49.5 & 49.5 \\
\hline 23 & 0.73 & 1.11 & 16.6 & 29.4 & 41.6 & 49.0 & 52.4 & 52.4 \\
\hline 16 & 0.90 & 1.10 & 25.3 & 44.7 & 63.3 & 74.5 & 79.5 & 79.5 \\
\hline 14 & 0.56 & 0.95 & 11.0 & 18.8 & 26.0 & 30.3 & 31.4 & 31.4 \\
\hline 12 & 0.43 & 0.94 & 6.6 & 11.2 & 15.4 & 17.9 & 18.5 & 18.5 \\
\hline 1 & 0.51 & 0.91 & 9.5 & 16.0 & 22.0 & 25.5 & 26.1 & 26.1 \\
\hline 19 & 0.49 & 0.91 & 8.9 & 14.9 & 20.4 & 23.6 & 24.2 & 24.2 \\
\hline 10 & 0.81 & 0.91 & 23.9 & 40.1 & 54.8 & 63.6 & 65.0 & 65.0 \\
\hline 2 & 0.49 & 0.88 & 8.8 & 14.6 & 19.8 & 22.9 & 23.2 & 23.2 \\
\hline 5 & 0.88 & 0.87 & 29.0 & 47.9 & 64.9 & 75.0 & 75.5 & 75.5 \\
\hline 3 & 0.55 & 0.80 & 11.8 & 18.9 & 25.0 & 28.6 & 27.9 & 27.9 \\
\hline 17 & 0.57 & 0.75 & 13.4 & 20.9 & 27.1 & 30.7 & 29.0 & 29.0 \\
\hline 15 & 0.46 & 0.75 & 8.6 & 13.4 & 17.3 & 19.5 & 18.3 & 18.3 \\
\hline 4 & 0.58 & 0.72 & 14.5 & 22.1 & 28.2 & 31.7 & 29.1 & 29.1 \\
\hline 26 & 0.23 & 0.33 & 2.7 & -0.5 & -5.6 & -8.9 & -16.8 & -16.9 \\
\hline 6 & 0.10 & 0.24 & 0.2 & -1.7 & -4.2 & -5.9 & -9.1 & -9.1 \\
\hline 7 & 0.16 & 0.17 & -1.1 & -12.7 & -27.6 & -37.3 & -54.6 & -54.6 \\
\hline 20 & 0.04 & 0.05 & -2.9 & -11.9 & -22.9 & -30.0 & -41.3 & -41.3 \\
\hline 8 & -0.12 & -0.33 & -3.0 & -7.9 & -13.5 & -17.0 & -21.8 & -21.8 \\
\hline 21 & -0.21 & -0.39 & -7.4 & -19.1 & -32.5 & -41.0 & -52.1 & -52.2 \\
\hline média & & & 12 & 23 & 33 & 38 & 40 & 40 \\
\hline$\beta_{c}$ & & & 0.2025 & 0.3431 & 0.4112 & 0.4363 & 0.4998 & $0.500 \mathrm{c}$ \\
\hline
\end{tabular}

Fonte: Resultados da pesquisa.

As Tabelas 3 e 4 ainda mostram a redução do risco de cada produtor como percentual do risco total. A redução média para os níveis de 95 a $120 \%$ é de $12,23,33,38$, 40 e $40 \%$, na Tabela 3 e de $5,21,31,31$, 31 e $31 \%$, na Tabela 4 . 
Um estudo da viabilidade de um programa de seguro agrícola baseado em um índice de produtividade regional em Castro (PR)

Retomando as Tabelas 1 e 2, observa-se que a redução média para os mesmos $\alpha_{i}$ 's foi de $4,6,10,15,4,16,6,16,6$ e $16,6 \%$, na Tabela 1 e de 2,6, 17,6, 32,9, 39,2, 39,7 e 39,7\%, na Tabela 2. Desta forma, nota-se que quanto maior o nível de agregação utilizado no design do contrato, maior será a redução do risco médio, dado o valor de $\alpha$.

Tabela 4. Coeficiente de correlação, $\beta_{i}$ e redução do risco e percentual da redução do risco, em relação ao risco total para a soja, ao nível de agregação cluster, em 40 fazendas, no período de 1994 a 2003.

\begin{tabular}{|c|c|c|c|c|c|c|c|c|}
\hline \multirow{2}{*}{ Num. } & \multirow{2}{*}{$\rho_{i}$} & \multirow{2}{*}{$\beta_{i}$} & \multicolumn{6}{|c|}{ Redução do Risco (\%) } \\
\hline & & & 0.95 & 1 & 1.05 & 1.1 & 1.15 & 1.2 \\
\hline 32 & 0.7672 & 2.3044 & 5.3 & 23.2 & 39.0 & 40.0 & 40.0 & 40.0 \\
\hline 33 & 0.6111 & 2.1674 & 3.6 & 15.5 & 25.8 & 26.5 & 26.5 & 26.5 \\
\hline 21 & 0.8517 & 2.1212 & 7.1 & 30.6 & 51.0 & 52.3 & 52.3 & 52.3 \\
\hline 12 & 0.8181 & 2.1038 & 6.6 & 28.4 & 47.3 & 48.5 & 48.5 & 48.5 \\
\hline 34 & 0.6814 & 1.8724 & 5.1 & 21.6 & 35.5 & 36.4 & 36.4 & 36.4 \\
\hline 25 & 0.6213 & 1.8484 & 4.3 & 18.2 & 29.8 & 30.5 & 30.5 & 30.5 \\
\hline 31 & 0.5040 & 1.7639 & 3.0 & 12.4 & 20.2 & 20.6 & 20.6 & 20.6 \\
\hline 9 & 0.4008 & 1.6912 & 1.9 & 8.1 & 13.1 & 13.4 & 13.4 & 13.4 \\
\hline 37 & 0.7549 & 1.6301 & 7.1 & 29.5 & 47.4 & 48.5 & 48.5 & 48.5 \\
\hline 38 & 0.7101 & 1.5687 & 6.5 & 26.9 & 42.9 & 43.8 & 43.8 & 43.8 \\
\hline 19 & 0.6381 & 1.4866 & 5.5 & 22.6 & 35.6 & 36.4 & 36.4 & 36.4 \\
\hline 15 & 0.3696 & 1.2988 & 2.1 & 8.3 & 12.7 & 12.9 & 12.9 & 12.9 \\
\hline 8 & 0.4867 & 1.1926 & 3.9 & 15.2 & 22.8 & 23.1 & 23.1 & 23.1 \\
\hline 10 & 0.4162 & 1.1511 & 3.0 & 11.4 & 16.8 & 17.0 & 17.0 & 17.0 \\
\hline 30 & 0.7368 & 1.1467 & 9.4 & 35.8 & 52.8 & 53.4 & 53.4 & 53.4 \\
\hline 17 & 0.7635 & 1.1180 & 10.3 & 39.0 & 57.0 & 57.6 & 57.6 & 57.6 \\
\hline 13 & 0.4256 & 1.0606 & 3.3 & 12.5 & 17.9 & 18.1 & 18.1 & 18.1 \\
\hline 18 & 0.5704 & 1.0425 & 6.1 & 22.6 & 32.2 & 32.5 & 32.5 & 32.5 \\
\hline 3 & 0.6323 & 0.9191 & 8.4 & 29.6 & 39.7 & 39.7 & 39.7 & 39.7 \\
\hline 39 & 0.6833 & 0.9068 & 9.9 & 34.8 & 46.3 & 46.2 & 46.2 & 46.2 \\
\hline 29 & 0.4208 & 0.7664 & 4.3 & 14.0 & 16.4 & 16.1 & 16.1 & 16.1 \\
\hline 36 & 0.5454 & 0.7587 & 7.3 & 23.5 & 27.3 & 26.7 & 26.7 & 26.7 \\
\hline 24 & 0.7429 & 0.7206 & 14.2 & 44.1 & 48.3 & 46.9 & 46.9 & 46.9 \\
\hline 11 & 0.6026 & 0.6873 & 9.7 & 29.2 & 30.0 & 28.8 & 28.8 & 28.8 \\
\hline 16 & 0.4580 & 0.6410 & 5.9 & 16.9 & 15.4 & 14.4 & 14.4 & 14.4 \\
\hline 14 & 0.2382 & 0.5169 & 1.9 & 4.2 & 1.3 & 0.7 & 0.7 & 0.7 \\
\hline 27 & 0.3197 & 0.5100 & 3.4 & 7.6 & 1.9 & 0.8 & 0.8 & 0.8 \\
\hline 28 & 0.5605 & 0.4517 & 11.6 & 20.1 & -10.0 & -14.9 & -14.9 & -14.9 \\
\hline 2 & 0.1995 & 0.3924 & 1.6 & 1.8 & -4.6 & -5.6 & -5.6 & -5.6 \\
\hline 26 & 0.2363 & 0.3055 & 2.6 & -1.4 & -20.8 & -23.3 & -23.3 & -23.3 \\
\hline 20 & 0.1450 & 0.2954 & 1.0 & -0.8 & -8.8 & -9.9 & -9.9 & -9.9 \\
\hline 7 & 0.1331 & 0.2816 & 0.8 & -1.1 & -8.8 & -9.8 & -9.8 & -9.8 \\
\hline 6 & 0.2779 & 0.2438 & 3.9 & -11.6 & -60.6 & -66.6 & -66.6 & -66.6 \\
\hline 22 & 0.1473 & 0.1734 & 1.0 & -11.8 & -43.4 & -47.1 & -47.1 & -47.1 \\
\hline 1 & 0.0760 & 0.1643 & 0.3 & -3.7 & -13.3 & -14.4 & -14.4 & -14.4 \\
\hline 23 & -0.2589 & -0.3059 & -6.5 & -48.0 & -109.5 & -115.4 & -115.4 & -115.4 \\
\hline 4 & -0.1087 & -0.3749 & -0.9 & -6.2 & -14.0 & -14.7 & -14.7 & -14.7 \\
\hline 40 & -0.2003 & -0.5951 & -1.7 & -11.1 & -23.6 & -24.8 & -24.8 & -24.8 \\
\hline 5 & -0.3207 & -0.6138 & -4.3 & -27.2 & -58.0 & -60.8 & -60.8 & -60.8 \\
\hline 35 & -0.3658 & -0.8325 & -4.0 & -23.7 & -49.1 & -51.4 & -51.4 & -51.4 \\
\hline média & & & 5.2 & 20.9 & 30.6 & 30.8 & 30.8 & 30.8 \\
\hline$\beta c$ & & & 0.11 & 0.33 & 0.49 & 0.50 & 0.50 & 0.50 \\
\hline
\end{tabular}

Fonte: Resultados da pesquisa. 
Novamente, neste caso, quanto maior $\beta_{i}$, maior será a redução do risco dado $\beta_{c}$ e a variabilidade da indenização, proveniente da aquisição de um contrato regional. Pela Tabela 4, acima, percebe-se que a variabilidade cluster é maior do que a variabilidade municipal. Neste caso, de acordo com a eq. (11), se o $\beta_{i}{ }^{2}$ for maior para todos os produtores quando o nível de agregação for cluster do que no caso municipal, então o componente sistêmico será maior para o primeiro nível de agregação.

Mas no caso em que o $\beta_{\mathrm{i}}{ }^{2}$ é menor, o risco sistêmico poderá ou não ser maior na agregação municipal. Isto de fato ocorre para 4 dos 26 produtores de milho e para 11 dos 40 produtores de soja.

\section{Conclusão}

O presente trabalho vem analisar a possibilidade de implementar um tipo de seguro baseado na produtividade regional. O seguro regional, se implantado na região de Castro, no Estado do Paraná, pode ser utilizado como um importante instrumento de administração do risco sistêmico pelos produtores.

Pelos resultados do trabalho, $81 \%$ e $92 \%$ dos produtores de milho se beneficiariam de alguma redução do risco sistêmico, pela aquisição deste tipo de seguro, respectivamente, para o nível de agregação municipal e cluster. No caso da soja, este percentual foi de 73 e $88 \%$, respectivamente. O nível de agregação cluster mostrou-se mais eficaz em reduzir o risco sistêmico, pois em termos médios, para todos os $\alpha$ 's, a redução do risco foi maior neste tipo de agregação.

Na análise da correlação, observou-se que a maioria dos produtores apresentou correlação positiva. Isto implica um menor ou maior grau de redução do risco sistêmico devido à aquisição deste tipo de contrato. Além disso, a correlação média entre a produtividade regional e individual mais do que dobrou quando considerado o nível de agregação cluster, para ambas as culturas.

Sendo assim, se tal seguro for implantado na região analisada, recomenda-se que o nível de agregação utilizado seja cluster, em acordo com as características homogêneas de solo, clima e tecnologia e também, de acordo com o zoneamento agrícola do Ministério da Agricultura.

Nota-se, porém, que a cobertura do risco não-sistêmico recai totalmen- 
te sobre os produtores. Uma forma de reduzir tal risco, seria a aquisição de um contrato de seguro que cobrisse eventuais reduções na produtividade individual e reduzisse as variações locais da produtividade agrícola.

\section{Referências bibliográficas}

AKERLOF, G.A. The market for "lemons": quality uncertainty and the market mechanism. Quarterly Journal of Economics, v.84, n.3, p.488500, Aug. 1970.

GOODWIN, B.K. An empirical analysis of the demand for multiple peril crop insurance. American Journal of Agricultural Economics, v.75, p.425-434, May 1993.

HALCROW, H. G. Actuarial structures for crop insurance. Journal of Farm Economics, v.31, n.3, p. 418-443, ago 1949.

HAZELL, P.; POMAREDA, C.; VALDÉS, A. Crop insurance for agricultural development. Baltimore: The Johns Hopkins University Press, 1986. $322 p$.

MAHUL, O. Optimum area yield crop insurance. American Journal of Agricultural Economics, v.81, n.1, p. 75-82, fev. 1999.

MIRANDA, M. J. Area-yield crop insurance reconsidered. American Journal of Agricultural Economics, v.73, n.2, p. 233-242, maio 1991.

MIRANDA, M. J.; GLAUBER, J. W. Systemic risk, reinsurance, and the failure of crop insurance markets. American Journal of Agricultural Economics, v.79, n.1, p. 206-215, fev 1997.

MIRANDA, M; SKEES, J.; HAZELL, P. Innovations in agricultural and natural disaster insurance for developing countries. Working paper, Dep. of Agr., Envir. and Development. Econ., The Ohio State University, 1999.

OZAKI, V.A. Métodos atuariais aplicados à determinação da taxa de prêmio de contratos de seguro agrícola: um estudo de caso. Piracicaba, 2005. 324p. Tese (Doutorado) - Escola Superior de Agricultura "Luiz de Queiroz", Universidade de São Paulo.

OZAKI, V.A.; SHIROTA, R. A experiência do seguro agrícola nos EUA: 
evolução e performance. Revista Brasileira de Risco e Seguro, v.1, n.2, Ago/Nov 2005. (no prelo)

QUINTÃO, C. Seguro agrícola: crescimento do mercado depende do governo. Gazeta Mercantil. São Paulo, 26 Set 2005. Agronegócio, B-12.

RAMASWAMI, B.; ROE, T. L. Aggregation in area-yield crop insurance: the linear additive model. American Journal of Agricultural Economics, v.86, n.2, p. 420-431, 2004.

ROSSETTI, L.A. Seguridade e zoneamento agrícola no Brasil: novos rumos. In: SIMPÓSIO INTERNACIONAL DE SECURIDADE E ZONEAMENTO AGRÍCOLA DO MERCOSUL, 1., Brasília, 1998. Anais. Brasília: Ministério da Agricultura e Abastecimento/CER/PROAGRO/GM, 1998. p.1-10.

ROSSETTI, L.A. Zoneamento agrícola em aplicações de crédito e securidade rural no Brasil: aspectos atuariais e de política agrícola. Revista Brasileira de Agrometeorologia, v.9, n.3, p.386-399, 2001.

SANTOS, C. G. O seguro rural. Revista do IRB, v.28, n.166, p.17-29, Dez. 1967.

SKEES, J. R.; BLACK, J. R.; BARNETT, B. J. Designing and rating an area yield crop insurance. American Journal of Agricultural Economics, v.79, n.2, p. 430-438, maio 1997.

SMITH, V. H.; CHOUINARD, H. H.; BAQUET, A. E. Almost ideal area yield crop insurance contracts. Agricultural and Resource Economics Review, v.23, n.1, abril 1994.

Recebido em março de 2005 e revisto em agosto de 2005 